\title{
斯-
}

\section{Motivaciones para el emprendimiento social}

\section{Esperanza García-Uceda}

Departamento de Dirección y Organización de Empresas

UNIVERSIDAD DE ZARAGOZA

mariola@unizar.es

Josefina L. MurilLo-Luna

Departamento de Dirección y Organización de Empresas

UNIVERSIDAD DE ZARAGOZA

jmurillo@unizar.es

Jesús Asín Lafuente

Departamento de Métodos Estadísticos

UNIVERSIDAD DE ZARAGOZA

jasin@unizar.es

Resumen: El objetivo de este trabajo consiste en identificar y comprender las motivaciones que subyacen en la decisión de optar por el emprendimiento social como modelo de negocio. Para ello, la metodología utilizada consiste en un análisis exploratorio estructurado en tres etapas. Primero, se aplica la metodología Delphi para 
identificar las principales motivaciones, con la colaboración de 20 expertos en emprendimiento social. Después, se analiza la valoración tanto de estos expertos como de un grupo de 21 emprendedores sociales, sobre la importancia de las motivaciones identificadas en esta decisión. Finalmente, se realiza un análisis comparativo de las valoraciones de ambos grupos para detectar diferencias significativas entre sus percepciones. Entre los resultados obtenidos destaca el consenso en cuanto a la fuerza de motivaciones relacionadas con la autorrealización, por cumplir un sueño movido por una extraordinaria pasión. Por el contrario, existe discrepancia entre expertos y emprendedores sociales sobre la incidencia de las motivaciones ideológicas, fundamentadas en una profunda conciencia social, y de las motivaciones económicas y de empleo.

Palabras clave: emprendimiento social, motivaciones, panel de expertos, emprendedores sociales, método Delphi.

\section{Motivations for social entrepreneurship}

Abstract: The purpose of this study is to identify and understand the reasons underpinning the decision to opt for social entrepreneurship as a business model. With this in mind, the methodology used involves carrying out an exploratory analysis structured in three stages. First, the Delphi method is applied to identify the main reasons motivating such a decision, with the aid of 20 experts in social entrepreneurship. Next, we analyse the assessment given both by these experts and a group of 21 social entrepreneurs, on the importance of the motivating factors identified in this decision. Finally, a comparative analysis of the assessments of each group is performed to detect any significant differences in their perceptions. Among the results obtained, most noteworthy is the consensus concerning the intensity of motivating factors relating to self-realisation, to fulfilling a dream driven by an extraordinary passion. On the other hand, there is discrepancy among experts and social entrepreneurs concerning the incidence of ideological motivating factors, based on a deep-rooted social awareness, along with economic and occupational factors.

Key words: social entrepreneurship, motivation, panel of experts, socialentrepreneurs, Delphi method. 


\section{Motivaciones}

para el

emprendimiento

social

Esperanza

García-Uceda

Josefina L.

Murillo-Luna

https://doi.org/10.26754/ojs_ais/ais.2019404203

Recibido: 02/10/2017

Aceptado: 12/04/2018

Jesús

Asín-Lafuente

\section{AGRADECIMIENTOS}

Agradecemos a todos los expertos y emprendedores sociales su valiosa contribución. Este trabajo ha recibido el apoyo financiero del Gobierno de Aragón y el Fondo Europeo de Desarrollo Regional (Grupos: CREVALOR, Modelos Estocásticos) y de los proyectos JIUZ-2016-SOC-09, ECO2016-77-P (AEI/FEDER, UE), MTM2017-83812-P.

\section{INTRODUCCIÓN}

Hasta la fecha no hay consenso acerca de la definición del concepto de emprendimiento social. Basándose en las aportaciones previas más ampliamente reconocidas en la literatura teórica, Dacin et al. (2010) explican que el emprendimiento social consiste en una iniciativa emprendedora cuya misión principal es la creación de valor social aportando soluciones a problemas sociales, tratando de lograr al mismo tiempo un complicado equilibrio con la creación de valor económico; cuestión esta última que, sin ser su misión principal, resulta crucial para este tipo de iniciativas y, por extensión, para la creación de valor social.

El emprendimiento social surge como alternativa al emprendimiento convencional, ante la incapacidad mostrada por los gobiernos, las empresas y la sociedad, en general, para tratar 
de dar solución a determinados problemas económicos, sociales y medioambientales, como el cambio climático, la desigualdad y la pobreza, las migraciones masivas o el terrorismo internacional, entre otros). (Shaw y Carter, 2007; Zahra, Rawhouser, Bhawe, Neubaum y Hayton, 2008; Dwivedi y Weerawardena, 2018).

La diferencia fundamental entre el emprendimiento convencional y el emprendimiento social reside en su misión. Mientras que el emprendimiento convencional persigue el retorno económico como objetivo prioritario (aunque pueda realizar además alguna contribución social), la misión principal del emprendimiento social consiste en generar un impacto positivo sobre un problema social, mediante la propuesta de soluciones innovadoras y de una forma sostenible, es decir, aplicando modelos económicos viables para alcanzar los objetivos sociales y/o medioambientales (Mair y Martí, 2006; Austin et al., 2006). En consecuencia, si bien el emprendimiento tradicional utiliza indicadores financieros para medir sus resultados, el emprendimiento social busca una combinación de resultados financieros y sociales (Boschee, 2008).

En las dos últimas décadas, el emprendimiento social ha experimentado un crecimiento exponencial (Pless, 2012; ReyMartí, Ribeiro-Soriano y Sánchez-García, 2015). Y su contribución a la economía es innegable. Según los datos publicados por la Confederación Empresarial Española de Economía Social (CEPES, 2018), la economía social en España está constituida por 42.140 empresas, cuya facturación representa el $10 \%$ del $\mathrm{PIB}$, lo que genera un total de 2.177.256 empleos directos e indirectos.

Nuestra atención en este trabajo se centra en estudio de los individuos a emprender socialmente. Precisamente las características de los emprendedores sociales individuales es uno de los cuatro factores clave identificados por Dacin, Dacin y Matear (2010) para definir el emprendimiento social, además del sector de actividad, los procesos y recursos utilizados y la misión principal y los resultados asociados con el emprendedor social. En efecto, muchas de las definiciones previas en la literatura se centran en las características de los emprendedores sociales, que determinan por qué algunas personas están predispuestas 
a interesarse en los asuntos sociales y otras no (Mair y Noboa, 2005) y tienden a resaltar las cualidades y el comportamiento de las personas relacionados con la motivación.

Autores como Austin, Stevenson y Wei-Skillern (2006) o Weerawardena y Mort (2006) destacan el interés del estudio de las motivaciones del emprendimiento social. Sin embargo, Dwivedi y Weerawardena (2018) ponen de manifiesto la falta de estudios sobre determinadas características del emprendimiento social, como las motivaciones que lo impulsan (Miller, Grimes, McMullen y Vogus, 2012; Fayolle y Liñán, 2014).

Teniendo en cuenta la valiosa contribución que el emprendimiento social hace a la economía (Thompson, 2002), nuestro objetivo con este trabajo es profundizar en el conocimiento de las motivaciones de las personas para optar por el emprendimiento social como modelo de negocio. El objetivo principal consiste en identificar y comprender las motivaciones individuales que existen detrás de la decisión de poner en marcha un proyecto de emprendimiento social. Para ello, se cuenta con la colaboración de un panel de expertos en emprendimiento social y un grupo de emprendedores sociales con quienes se reflexiona sobre estas cuestiones. La metodología empleada en la investigación para la identificación y valoración de las motivaciones es el método subjetivo de juicio de experto. Esta es una estrategia bastante habitual para ampliar el conocimiento de contenidos y temas que son difíciles, complejos, nuevos o que no se han estudiado con frecuencia (Lannoy y Procaccia, 2001) y constituyen la base del método Delphi (Romero et al., 2012). Para el análisis comparativo de las valoraciones de ambos grupos, se lleva a cabo análisis estadísticos con objeto de identificar posibles diferencias significativas entre sus valoraciones.

Con este objetivo en mente, la estructura del trabajo es la siguiente. Primero, se revisa la literatura para proponer un marco teórico que identifique las motivaciones individuales que hay detrás del emprendimiento social. En segundo lugar, se describe la metodología que se utiliza. En tercer lugar, se presentan los resultados más significativos. Por último, se resumen las principales conclusiones, se comentan las limitaciones del estudio y se sugieren posibles líneas de investigación futuras. 


\section{MARCO TEÓRICO SOBRE MOTIVACIONES Y EMPRENDIMIENTO SOCIAL}

El emprendimiento social y el emprendimiento convencional tienen algunos rasgos en común. Ambos identifican oportunidades en el entorno inmediato y buscan recursos para aprovecharlos (Cools y Vermeulen, 2008). Por lo tanto, comparten características significativas (Steinerowski, Jack y Farmer, 2008) y, sin duda, también tienen algunas motivaciones en común. En consecuencia, se debería comenzar por revisar la literatura existente sobre motivaciones empresariales en general (Dacin et al., 2010).

La motivación de las personas se refiere a las razones que explican por qué se esfuerzan por realizar y luego llevar a cabo una acción (Latham y Pinder, 2005) y desempeña un importante papel en la creación de nuevas organizaciones, en general, dado que influye en la toma de decisiones (Segal, Borgia y Schoenfeld, 2005).

En el espíritu del emprendimiento, las teorías de motivación han evolucionado de teorías de contenido a teorías de proceso (Segal et al., 2005). Este trabajo se centra en explicar las necesidades u objetivos que llevan a una persona elegir el emprendimiento social como modelo de negocio sostenible, sin intentar describir cómo se produce un determinado comportamiento. Por ello, se adopta la perspectiva de las teorías de contenido.

Dentro del marco de las teorías de contenido, varios autores (desde Gilad y Levine (1986) hasta Kirkwood y Walton (2010) más recientemente) se apoyan en la teoría de presión y de atracción para entender las motivaciones empresariales. Los factores de presión consisten en fuerzas externas negativas que empujan a los individuos hacia el espíritu empresarial. Por otro lado, los factores de atracción son aspectos que atraen a las personas a poner en marcha un negocio (Kirkwood, 2009). Los resultados de diferentes investigaciones evidencian que los factores de atracción predominan sobre los factores de presión para explicar la decisión de convertirse en empresario (Orhan y Scott, 2001; Noorderhaven, Thurik, Wennekers y van Stel, 2004) y la mayor probabilidad de éxito de los proyectos promovidos por estos factores de atracción (Amit y Muller, 1995). 
Según Kirkwood y Walton (2010), hay cuatro motivaciones principales que conducen a la iniciativa empresarial: el deseo de independencia, motivaciones monetarias, aspectos relacionados con el trabajo (como la insatisfacción, inestabilidad, deseo de una mayor flexibilidad o proyección profesional, dificultades para encontrar un trabajo, etcétera) y cuestiones familiares (básicamente dificultades para conciliar trabajo y vida familiar). Los dos primeros son factores de atracción, mientras que los dos últimos son factores de presión. Otros autores que añaden motivaciones adicionales que completan el conjunto de factores de atracción, tales como la voluntad de crear e innovar, la propensión a asumir riesgos, el reconocimiento de una oportunidad, el reconocimiento personal o la autorealización (Mair y Noboa, 2005; Mair y Martí, 2006; Hall, Miller y Millar, 2012; Marques, Ferreira, Ferreira y Lages, 2012; Braga, Proença y Ferreira, 2014).

Pero el conocimiento actual de las motivaciones empresariales convencionales no es suficiente para explicar las motivaciones empresariales sociales. Más aún, en algunos casos es inadecuado (Braga et al., 2014). La preocupación por la sociedad es precisamente lo que diferencia el emprendimiento social del emprendimiento convencional y, por lo tanto, el marco teórico sobre motivaciones emprendedoras sociales también debe considerar las emociones prosociales. Estas motivaciones explicarían las decisiones que llevan a acciones dirigidas al bienestar del grupo, incluso a expensas de los intereses individuales (Miller et al., 2012).

En consecuencia, otras motivaciones son intrínsecas y distintivas del emprendimiento social (Steinerowski et al., 2008). La mayoría de ellas son factores de atracción (Braga et al., 2014) como, por ejemplo, el altruismo y la pasión. El altruismo hace referencia a la misión desinteresada de buscar el bienestar de la sociedad o el entorno que la rodea (Dacin et al., 2010), el deseo de trabajar por el bienestar de la sociedad y la preocupación por hacer felices a los demás (Braga et al., 2014). La pasión implica una motivación intrínseca por hacer las cosas de forma diferente, actuando de una manera socialmente responsable, un sentimiento de disconformidad con el status quo acompañado de una fuerte lealtad a los valores y la filosofía propios y un intenso deseo de cambiar la sociedad, así como la creencia de 
que es posible convencer a otros de que hagan lo mismo (Prabhu, 1999; Steinerowski et al., 2008).

Miller et al. (2012) también hacen referencia a emociones como la compasión. Cuando el espíritu empresarial social se conceptualizó por primera vez, los académicos argumentaron que la decisión de poner en marcha este tipo de empresa estaba motivada sustancialmente por un sentimiento de compasión por los demás (Dees, 1998; 2007). La compasión se caracteriza por la orientación hacia los demás y una conexión emocional que une a una persona con una comunidad que sufre (Goetz, Keltner y Simon-Thomas, 2010). Puede actuar como un potente factor de motivación que lleva a los individuos a tratar de persuadir a otras personas para que alivien el sufrimiento de los demás (Omoto, Malsch y Barraza, 2009), complementando las motivaciones tradicionales centradas en el interés personal y alentando así el emprendimiento social (Miller et al., 2012).

Por último, existen motivaciones cuya clasificación como factores de atracción o presión no es tan clara o excluyente. Se trata de la influencia de modelos a seguir (familia, amigos o grupos que pueden haber estado implicados en algún tipo de iniciativa de emprendimiento social), o experiencias previas, por ejemplo, el voluntariado asociado con los valores de las personas y un sentimiento de utilidad (Braga et al., 2014).

La Figura 1 muestra las aportaciones de la literatura teórica sobre motivaciones que explican el emprendimiento social, que incluyen motivaciones compartidas con el emprendimiento convencional y las motivaciones propias del emprendimiento social.

A partir de esta revisión de la literatura teórica, se pueden plantear las siguientes proposiciones sobre las motivaciones para el emprendimiento social para su futuro estudio:

Proposición 1: Algunas de las motivaciones para el emprendimiento social coinciden con las motivaciones para emprendimiento convencional, pero también existen otras motivaciones específicas o propias del emprendimiento social.

Proposición 2: Las motivaciones para el emprendimiento social que representan factores de atracción predominan sobre aquéllas que constituyen factores de presión. 


\section{Figura 1 \\ Motivaciones y emprendimiento social}

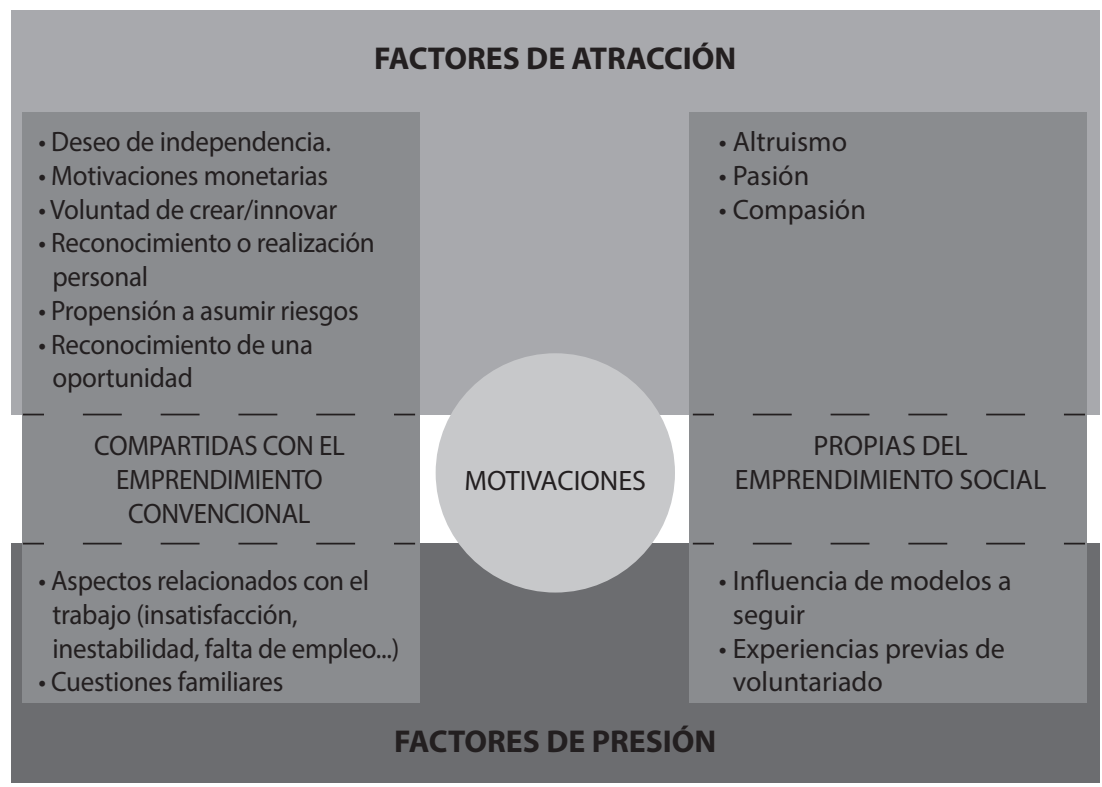

Fuente: Elaboración propia.

\section{METODOLOGÍA}

En este estudio, se realiza un análisis exploratorio estructurado en tres etapas. Para implementar la primera y la segunda etapa se utiliza el método Delphi. Este método consiste en un proceso sistemático e iterativo encaminado a la obtención de las opiniones $\mathrm{y}$, a ser posible, el consenso estadístico, de un grupo de expertos anónimos a los que se aplica una serie de cuestionarios con retroalimentación controlada (Landeta, 2002). Las respuestas de los panelistas son analizadas cualitativa y cuantitativamente, a través del tratamiento de medianas y los consecuentes intervalos de confianza. Se considera que entre los panelistas se ha alcanzado el mayor consenso posible, a través de la medida de la varianza en sus respuestas de las diferentes rondas (Rowe y Wright, 1999). García-Ruiz y LenaAcebo (2018) trabajan con este procedimiento para obtener una 
herramienta capaz de obtener información útil para caracterizar cuantitativamente el fenómeno de las actividades colaborativas tecnológicas.

En la primera etapa, con la colaboración de un panel de expertos en emprendimiento social, se diseña un cuestionario dirigido a identificar las principales motivaciones que actúan como un estímulo para poner en marcha un proyecto de emprendimiento social. En la segunda etapa, se incorpora al estudio a un grupo de emprendedores sociales y se solicita tanto al panel de expertos como al panel de emprendedores sociales su valoración sobre la incidencia de las motivaciones identificadas en la etapa anterior.

En la tercera etapa se utiliza la prueba $U$ de Mann-Whitney para realizar un análisis comparativo de las respuestas de ambos grupos, expertos y emprendedores sociales. Su principal utilidad es que permite estudiar y reflexionar sobre la posible existencia de diferencias significativas entre las percepciones de ambos grupos.

Para llevar a cabo la selección de los expertos se siguen las recomendaciones de autores como García y Fernández (2008), esto es, por su vinculación con el problema, experiencia profesional, cualidades personales para participar en la investigación y pericia profesional. Con relación al número de expertos, Landeta (2002), por ejemplo, recomienda entre 7 y 30 y para García y Fernández (2008) el intervalo debe situarse entre 15 y 25. Siguiendo estas orientaciones, en enero de 2017 se reúne un grupo de 23 expertos, 15 hombres y 8 mujeres, para diseñar el cuestionario. De los 23 expertos contactados inicialmente por correo electrónico para invitarlos a participar en la investigación, 20 finalmente aceptan colaborar. Todos ellos desarrollan su actividad en el territorio español. Algunos ostentan cargos de responsabilidad en entidades sociales privadas (15\%) -como Fundación Tomillo, Cáritas y Nittúa-, otros son especialistas en investigación académica sobre emprendimiento social y actividades de transferencia (25\%) -del Programa Spin UP, Universidad de Zaragoza, Centro Europeo de Empresas e Innovación de Aragón (CEEI), Instituto CIRCE, Cátedra Emprendedores, Universidad de Cádiz, ToBeinn Network- y, por último, también participan consultores de emprendimiento social para 
organizaciones privadas y públicas (60\%) -de Zaragoza Activa y Fomento Empresarial (Ayuntamiento de Zaragoza), El HuecoSoria, CEOE, Stone Soup Consulting, Fundación Zaragoza Ciudad el conocimiento, Cámara de Comercio Zaragoza, Fundación CREAS, AV/Asesores, Instituto Aragonés de Fomento (IAF), Emoziona y EMES International Research Network.

De acuerdo con Fernández-Ballesteros (1995), primero se hace una pregunta abierta a estos 20 expertos, a saber: ¿Cuáles son las principales motivaciones para que una persona decida optar por el emprendimiento social?

Sus respuestas nos llevan a identificar inicialmente 62 motivaciones. Se considera que debería realizarse un análisis previo del contenido, dado el alto número de elementos y, especialmente, al observar diferentes grados de diferenciación en las respuestas. A continuación, en coherencia con la revisión de literatura teórica realizada en el apartado anterior, se infieren dimensiones teóricas. Así es como se llega a un cuestionario cerrado que incluye 23 motivaciones agrupadas en las siguientes cinco dimensiones: motivaciones ideológicas, motivaciones de realización personal, motivaciones financieras y de empleo, motivaciones de desarrollo sostenible y motivaciones de enlace (Tabla 1). 


\section{Tabla 1}

\section{Principales motivaciones para el emprendimiento social}

\begin{tabular}{|c|c|c|c|}
\hline \multirow{8}{*}{ 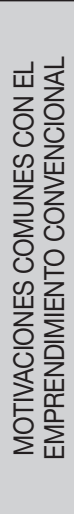 } & \multirow{5}{*}{$\begin{array}{l}\text { Económicas } \\
\text { y de empleo }\end{array}$} & $\begin{array}{l}\text {-Realizar una actividad comercial y empresarial responsable, replicable y } \\
\text { escalable (de lo local a lo internacional). }\end{array}$ & \multirow{5}{*}{ 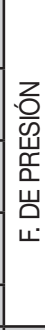 } \\
\hline & & $\begin{array}{l}\text {-Autoempleo en su afición liderando su iniciativa o simplemente ganarse la } \\
\text { vida trabajando en lo suyo. }\end{array}$ & \\
\hline & & $\begin{array}{c}\text {-Generar empleo: Crear puestos de trabajo adecuados (de calidad) y } \\
\text { sostenibles (estables). }\end{array}$ & \\
\hline & & $\begin{array}{l}\text {-Mejores costes. Obtener una ventaja competitiva en los costes por bonifi- } \\
\text { caciones fiscales y seguridad social. }\end{array}$ & \\
\hline & & -Obtener acceso a determinadas subvenciones y concursos públicos. & \\
\hline & \multirow{3}{*}{$\begin{array}{l}\text { De realización } \\
\text { personal }\end{array}$} & -Reconocimiento social, personal y de la labor realizada. & \multirow{10}{*}{ 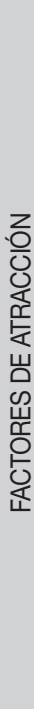 } \\
\hline & & $\begin{array}{c}\text {-Autorrealización y Autoestima. Realización personal liderando un sueño } \\
\text { de una manera adecuada a su forma de pensar. }\end{array}$ & \\
\hline & & $\begin{array}{l}\text { - Capacidad emprendedora y pasión por la misión de su proyecto } \\
\text { empresarial. }\end{array}$ & \\
\hline \multirow{15}{*}{ 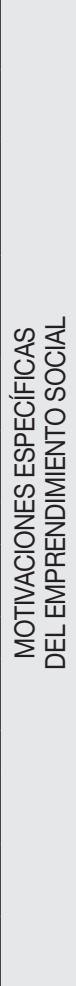 } & \multirow{6}{*}{ Ideológicas } & $\begin{array}{c}\text {-Convicción ideológica. Convencimiento de la primacía de las personas } \\
\text { sobre las estructuras productivas y el capital económico. }\end{array}$ & \\
\hline & & $\begin{array}{c}\text { - Concienciación política (militancia política no partidista) vinculada a lo } \\
\text { social. }\end{array}$ & \\
\hline & & $\begin{array}{l}\text { - Solidaridad o Altruismo. Búsqueda de una rentabilidad emocional: sentir } \\
\text { que el trabajo proporciona una mejora a la sociedad. }\end{array}$ & \\
\hline & & - Conciencia de justicia social. Búsqueda de una sociedad más equitativa. & \\
\hline & & $\begin{array}{l}\text { - Responsabilidad social. Conciencia de la existencia de una necesidad } \\
\text { social o medioambiental que el sistema no atiende o no lo hace de forma } \\
\text { eficaz y/o eficiente. }\end{array}$ & \\
\hline & & $\begin{array}{l}\text { - Convicción de cambio y transformación social. Activismo sobre } \\
\text { ineficiencias sociales. }\end{array}$ & \\
\hline & \multirow{4}{*}{$\begin{array}{l}\text { De desarrollo } \\
\text { sostenible }\end{array}$} & $\begin{array}{l}\text { - Creación de valor social con sentido práctico y valor económico } \\
\text { sostenible. }\end{array}$ & \\
\hline & & - Inclusión social. Integración de personas marginadas. & \\
\hline & & $\begin{array}{l}\text { - Favorecer un trabajo colaborativo y no competitivo. Las cosas se pueden } \\
\text { hacer de una forma diferente. }\end{array}$ & \multirow{7}{*}{ 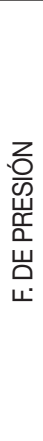 } \\
\hline & & - Seguir una tendencia social de moda. & \\
\hline & \multirow{5}{*}{$\begin{array}{c}\text { Por } \\
\text { vinculación }\end{array}$} & $\begin{array}{l}\text { - Vinculación con espacios urbanos con cronificación de situaciones gene- } \\
\text { radoras de riesgos de exclusión. Involucrarte con la realidad del entorno. }\end{array}$ & \\
\hline & & - Vinculación o implicación personal o familiar con necesidades sociales. & \\
\hline & & $\begin{array}{l}\text { - Vinculación con entidades de carácter social que desean desarrollar } \\
\text { proyectos en el marco empresarial social. }\end{array}$ & \\
\hline & & $\begin{array}{l}\text { - Iniciativas desde lo público que se transfiere para su ejecución a } \\
\text { promotores particulares de componente social. }\end{array}$ & \\
\hline & & $\begin{array}{c}\text { - Vinculación trasversal de lo social con conceptos medio ambientales, } \\
\text { desarrollo cultural o educativos. }\end{array}$ & \\
\hline
\end{tabular}

Fuente: Elaboración propia. 
En la segunda etapa se incorpora al estudio un grupo o panel de emprendedores sociales. Todos están trabajando en ese momento en proyectos de emprendimiento social, creados en el marco de diferentes ediciones del Programa de Emprendimiento Social en Aragón, organizado por el Instituto Aragonés de Fomento y el Instituto Aragonés de Servicios Sociales, dos organizaciones públicas del Gobierno de Aragón (España). Esta vez se contacta con 30 personas, también por correo electrónico. Finalmente, 21 emprendedores sociales colaboran en el estudio, nueve hombres y 12 mujeres.

A continuación, se proporciona a los 20 expertos y a los 21 emprendedores sociales el cuestionario cerrado obtenido en la primera etapa y se les pide su valoración de la importancia de cada una de las motivaciones en el cuestionario, utilizando una escala de 0 a 10 puntos.

Como se pretende lograr un consenso estadístico en cada grupo o panel, en una segunda ronda se informa a cada experto y a cada emprendedor social de la medida en que sus calificaciones coinciden con las calificaciones promedio de su grupo, al tiempo que se garantiza que todos los miembros del grupo permanezcan en el anonimato, para evitar que la opinión de algunas personas conocidas pueda influir en las calificaciones de otros (Martínez, 2003). A todos se les da la opción de cambiar sus respuestas, cuando lo consideren adecuado. Cuando deciden mantener sus valoraciones y éstas difieren de la calificación promedio en su grupo, se les pide que expliquen sus razones para ello.

Se utiliza la Proporción de expertos para analizar de manera descriptiva el consenso entre las opiniones de los miembros de cada grupo. Éste se alcanza cuando ninguno de los expertos modifica su respuesta a una determinada pregunta (Scheibe et al., 1975). Se descubre que el valor medio de las últimas respuestas dista entre $[-0.5$ y +0.5$]$ del valor medio de sus valoraciones anteriores. Esto permite concluir que más de la mitad de las valoraciones de los panelistas de cada grupo no han cambiado sustancialmente (específicamente más del $65 \%$ de las motivaciones en el grupo de expertos y el $100 \%$ de las motivaciones en el grupo de emprendedores sociales). Estos resultados evidencian la considerable convergencia entre las calificaciones de los miembros 
de cada grupo, especialmente en el caso de los emprendedores sociales.

Después, para conocer el grado de estabilidad en las respuestas sucesivas de los miembros de ambos paneles, se utiliza la prueba de Wilcoxon, cuya hipótesis nula es la falta de cambios significativos en las calificaciones. Esta es una prueba no paramétrica recomendable para muestras relacionadas, cuando no podemos asumir la condición de normalidad de los datos para tamaños de muestra reducidos y en diseños en dos fases. Los resultados de este análisis muestran una falta de evidencia de cambios significativos en las respuestas de los miembros de ambos grupos en relación con el $100 \%$ de las motivaciones (valor de p> $0,05)$. Por lo tanto, no es necesaria una tercera ronda (FernándezBallesteros, 1995).

En la tercera etapa, se realiza un análisis comparativo de las calificaciones promedio en los dos paneles, expertos y emprendedores sociales, para estudiar la posible existencia de diferencias significativas entre ellos. En este sentido, cabe señalar que la disponibilidad de respuestas cuantitativas se aprovecha para trabajar con contrastes estadísticos que presentan más potencia que los basados en información binaria, tal como analizan García-Ruiz y Lena-Acebo (2018). Primero, se analizan las desviaciones de la normalidad en los resultados utilizando la estadística Anderson Darling (AD), que confirma que los resultados no siguen una distribución normal en ninguno de los grupos. Luego, para comparar las distribuciones de los resultados de ambos paneles, se utiliza la prueba $U$ de Mann-Whitney $\left(H_{0}=\right.$ la mediana de las respuestas de ambos grupos es la misma). Esta es una prueba no paramétrica para muestras independientes que permite analizar las diferencias entre las calificaciones de los expertos y los emprendedores sociales con varios niveles de significación (5\%, 1\% y $0,1 \%$ ) para cada una de las 23 motivaciones analizadas.

\section{RESULTADOS Y DISCUSIÓN}

A continuación, se presentan dos subapartados que contienen los principales resultados, que también se ilustran en la Figura 2. El primero se centra en las valoraciones consensuadas en cada panel, es decir, los expertos y los emprendedores 
sociales. El segundo se centra en el análisis comparativo de las calificaciones de estos dos paneles de especialistas en emprendimiento social.

\section{Valoraciones de los expertos y de los emprendedores sociales}

\section{Valoraciones del panel de expertos}

La Figura 2 muestra las valoraciones medias alcanzadas por consenso entre los expertos (en verde - EXP). Como se puede observar, en su opinión, las motivaciones más importantes se derivan de la dimensión "Motivaciones de realización personal": 9. Capacidad emprendedora y pasión por la misión [...] (8.5) y 8. Autorrealización y autoestima. [...] (8.4). Además, los expertos destacan otras variables en la dimensión "Motivaciones ideológicas" como 5. Responsabilidad social. [...] (8.1), la 1. Convicción ideológica. [...] (7.7), 6. Convicción de cambio y transformación social. [...] (7.4) y 4. Conciencia de justicia social. [...] (7.4). Otras motivaciones con impacto destacable son 20. Vinculación o implicación personal o familiar con necesidades sociales [...] (7.3) y 16. Inclusión social. [...] (7.2). Este rango de impacto incluye 3. Solidaridad o Altruismo. [...] (7.1) y 15. Creación de valor social con sentido práctico y valor económico sostenible (7.1). 


\section{Figura 2}

Valoraciones medias de expertos y emprendedores sociales sobre las motivaciones para el emprendimiento social

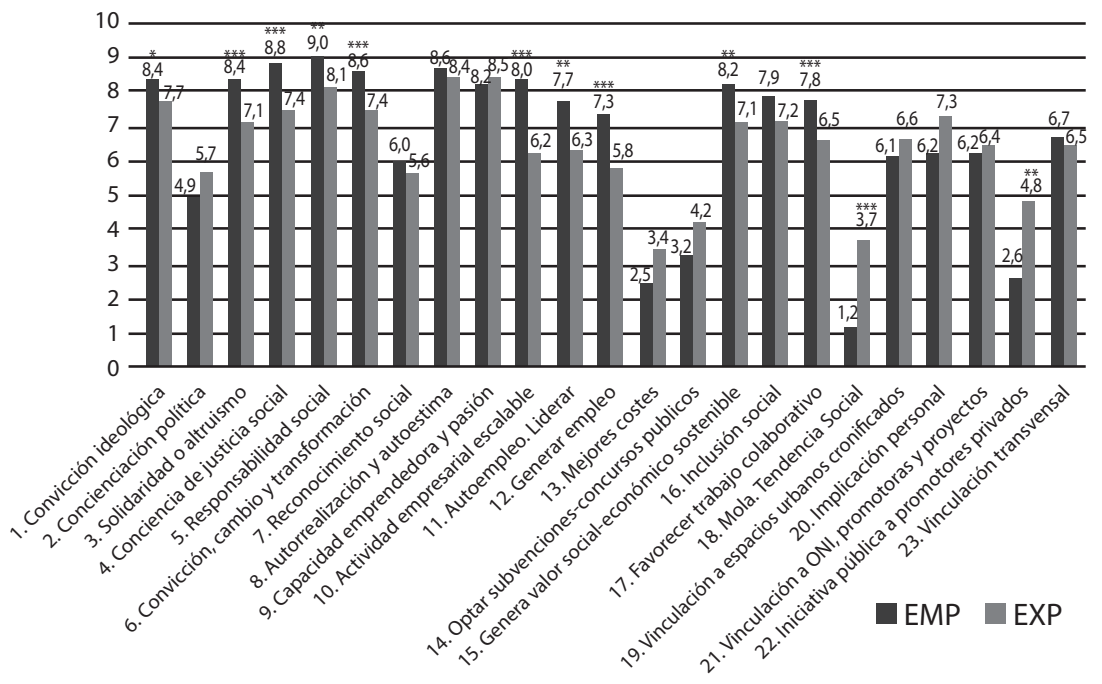

Se añaden los símbolos * ${ }^{* *} \mathrm{y}^{* * *}$ para indicar cuándo el test de Mann-Whitney ha resultado significativo a nivel $5 \%, 1 \%$ y $0.1 \%$, respectivamente.

${ }^{*}=(0,01<p$-valor $<0,05)$ (significación al 5\%) Apenas hay diferencia.

${ }^{* *}=(0,001<p$-valor $\leq 0,01)$ (significación al 1\%) Hay diferencia.

${ }^{* \star *}=(p-v a l o r \leq 0,001)$ (significación al $\left.0,1 \%\right)$ Existe una clara diferencia.

Ninguna de las siguientes motivaciones recibió una valoración inferior a 6 de todo el grupo de expertos: 19. Vinculación con espacios urbanos con cronificación de situaciones generadoras de riesgos de exclusión. [...] (6.6), 23. Vinculación trasversal de lo social con conceptos medioambientales, desarrollo cultural o educativos (6.5) y 21. Vinculación con entidades de carácter social que desean desarrollar proyectos en el marco empresarial social (6.4), y aquellas agrupadas como "Motivaciones económicas y de empleo": 10. Realizar una actividad comercial y empresarial replicable y escalable [...] (6.2) y 11. Autoempleo en su afición liderando su iniciativa [...] (6.3). Destaca también el estímulo del desarrollo sostenible. Según los expertos, esto corresponde a 
un sentimiento generalizado de que las personas pueden crear riqueza de manera diferente, esto es, 17. Favorecer un trabajo colaborativo y no competitivo. [...] (6.5).

En contraste, algunas motivaciones tienen menos incidencia según los expertos. Los emprendedores sociales han elegido este camino debido a sus convicciones, por lo que la mayoría de las motivaciones en la dimensión 'Motivaciones económicas y de empleo' se perciben como fuerzas con menos impacto: 13. Mejores costes. [...] (3.4) y 18. Seguir una tendencia social de moda (3.7). Otro aspecto de poco interés es 14. Optar a determinadas subvenciones y concursos públicos (4.2) o desarrollar proyectos de otros, alejados de sus sueños, esto es, 22. Iniciativas desde lo público que se transfiere para su ejecución a promotores particulares de componente social (4.8).

\section{Valoraciones del panel de emprendedores sociales}

La Figura 2 también muestra las valoraciones medias alcanzadas por consenso entre los emprendedores sociales (en azul - EMP). Como se puede observar, las motivaciones más importantes para los emprendedores sociales forman la dimensión "Motivaciones ideológicas": 5. Responsabilidad social. [...] (9.0), 4. Conciencia de justicia social. [...] (8.8), 6. Convicción de cambio y transformación social [...] (8.6) y 1. Convicción ideológica. $[. .].(8,4)$. La búsqueda y el logro de valores éticos y sociales predomina sobre el beneficio en el emprendimiento social, 8. Autorrealización y Autoestima. [...] (8.6) y 15. Creación de valor social con sentido práctico y valor económico sostenible (8.2). En este sentido, podemos observar que los emprendedores sociales otorgan una importancia considerable a los incentivos interiorizados positivos, como 9. Capacidad emprendedora y pasión por la misión [...] (8.2). Hacer el bien social está en el ADN de los emprendedores sociales. Es por eso que sus desafíos están principalmente relacionados con 16. Inclusión social. [...] (7.9).

Otras motivaciones con una incidencia intermedia son "Motivaciones económicas y de empleo": 10. Realizar una actividad comercial y empresarial replicable y escalable [...] (8.0), 11. Autoempleo en su afición [...] (7.7) y 12. Generar empleo. [...] (7.3), en línea con el estímulo del desarrollo sostenible que corresponde a 
un sentimiento generalizado de que las personas pueden crear riqueza de manera diferente, buscando un desarrollo sostenible, esto es, 17. Favoreciendo un trabajo colaborativo y no competitivo. [...] (7.8).

Finalmente, hay otra serie de ítems que obtienen unas valoraciones muy bajas, por debajo del 5, en la opinión de nuestro panel de emprendedores. En primer lugar, de poco interés, incluso claramente despreciable es 18. Seguir una tendencia social. [...] (1.2). Optar por el emprendimiento social no tiene nada que ver con la frivolidad de la moda. También destacan otras motivaciones en la dimensión "Motivaciones económicas y de empleo", que confirman que las personas se convierten en emprendedores sociales debido a su convicción; son personas de principios que no se dirigen a este sector porque aparentemente hay ayuda financiera disponible o para implementar proyectos de otros que no coinciden con sus sueños: 14. Optar a determinadas subvenciones y concursos públicos (3.2) y 22. Iniciativas desde lo público que se transfiere para su ejecución a promotores particulares de componente social (2.6). Los emprendedores sociales no buscan alcanzar 13. Mejores costes. [...] (2.5).

\section{Análisis comparativo de las valoraciones de ambos paneles}

La Figura 2 también recoge los resultados del análisis comparativo de las valoraciones de ambos paneles e identifica aquellos ítems en los que existen diferencias significativas entre ambos paneles. Se indican con símbolos su nivel de significación (5\%, *; $\left.1 \%,{ }^{* *} ; 0,1 \%,{ }^{* * *}\right)$.

Los resultados muestran, en primer lugar, un claro acuerdo entre los miembros de ambos paneles sobre 10 de las motivaciones analizadas. Ninguno de ellos presenta diferencias significativas, en otras palabras, las opiniones de los panelistas muestran un claro acuerdo con respecto al impulso positivo de determinadas motivaciones para el emprendimiento social. Destacan especialmente que las dos variables con mayor atracción se agrupan en la dimensión 'Motivaciones de realización personal': 8. Autorrealización y Autoestima, [...] y 9. Capacidad emprendedora y pasión por la misión, [...]. 
Con relación a las motivaciones ideológicas, motores del cambio, se encuentran discrepancias con varios niveles de importancia entre ambos paneles: 4. Conciencia de justicia social, [...], 6. Convicción de cambio y transformación social, [...], y 3. Solidaridad o Altruismo (con un nivel de significación del $0,1 \%$, ${ }^{* * *}$ ), 5. Responsabilidad social, [...] (con un nivel de significación del $1 \%,{ }^{\star *}$ ) y 1 . Convicción ideológica, [...] (con un nivel de significación del $\left.5 \%,{ }^{*}\right)$. Los emprendedores sociales conceden a estas motivaciones las máximas puntuaciones medias, mientras que los expertos no reconocen en estos aspectos los mayores impulsos positivos. En consecuencia, para los emprendedores sociales la pasión con la que administran sus recursos personales es un activo valioso para la realización personal, para buscar un impacto social positivo de su negocio que vaya más allá de obtener una ganancia económica. Están absolutamente decididos y convencidos de que pueden superar todas las resistencias para producir un cambio positivo en la sociedad, al preocuparse por mejorar el bienestar de los demás y su entorno y hacer de su sueño una profesión.

En cuanto a las motivaciones relacionadas con el empleo y la sostenibilidad de los proyectos, una vez más la valoración media de los emprendedores sociales es superior, en al menos 1,4 puntos, a la de los expertos, por lo que perciben que planifican actividades comerciales capaces de 12. Generar empleo. [...] y 10. Realizar una actividad comercial y empresarial replicable y escalable [...] (ambas con un nivel del 0,1\%, ${ }^{* *}$ ). En este sentido, los emprendedores sociales se sienten atraídos por esta opción 11. Autoempleo en su afición [...] (con un nivel del 1\%, ${ }^{* \star}$ ) y generar riqueza de otra forma, esto es, 15 . Crear valor social con sentido práctico y valor económico sostenible (con un nivel del $1 \%,{ }^{* *}$ ) y 17. Favorecer un trabajo colaborativo y no competitivo (con un nivel del $\left.0,1 \%,{ }^{* * *}\right)$.

Ocurre lo contrario en el caso de las motivaciones por vinculación. Los emprendedores sociales muestran valoraciones medias muy por debajo de las opiniones de los expertos, alrededor de 1 punto en el caso de 20. Vinculación personal o familiar, [...] (con un nivel del $5 \%$, *) y una diferencia de alrededor de 2 puntos para 22. Iniciativas desde lo público que se transfiere para su ejecución a promotores particulares de componente social (con un nivel del $\left.1 \%,{ }^{\star \star}\right)$. No obstante, los dos paneles (en especial el formado por 
los emprendedores sociales) apenas perciben presión por estos factores, es decir, su poder de motivación es débil. También vale la pena destacar el resultado obtenido con la motivación 18. Seguir una tendencia social de moda. Si bien los emprendedores sociales afirman que no tiene relevancia $(1,2)$, el panel de expertos otorga una calificación ligeramente más alta $(3,7)$, aunque la incidencia aún es baja.

Finalmente, podemos observar que el rango de valoraciones para las 13 motivaciones que muestran discrepancias significativas es más amplio en el panel de los emprendedores sociales. Esto puede indicar que es más probable que su criterio distinga entre fuerzas de mayor impulso (por ejemplo, la 5. Responsabilidad social, [...], con una puntuación media de 9) y lo despreciable (18. Seguir una tendencia social de moda, con una puntuación media de 1,2). Los emprendedores sociales perciben las motivaciones agrupadas como ideológicas, de desarrollo sostenible, económicas y de empleo en torno a 1 punto o incluso 2 puntos por encima de la fuerza que les otorga el panel de expertos. Estos resultados podrían resaltar el optimismo y la audacia innata de los emprendedores sociales, alimentados por convicciones y creencias más idealistas y por las emociones y sensaciones de satisfacción que se derivan de realizar su sueño, en comparación con la objetividad de los expertos y el razonamiento crítico que carece de un vínculo directo con el proyecto y el entorno del emprendedor social.

En definitiva, hay diferencias significativas entre las valoraciones de los paneles de expertos en emprendimiento social y los emprendedores sociales. Esto pone de manifiesto la necesidad de un estudio más profundo sobre las percepciones de las diferentes partes implicadas en el fenómeno del emprendimiento social.

En coherencia con otros autores, como Dacin et al. (2010), los resultados obtenidos muestran que, en efecto, algunas de las principales motivaciones para el emprendimiento social son comunes con las motivaciones identificadas en la literatura para el emprendimiento convencional, ofreciendo así unos primeros indicios coincidentes con el argumento de la Proposición 1. Ahora bien, los resultados indican que las motivaciones más importantes forman parte tanto de los factores de atracción (como, por 
ejemplo, las motivaciones ideológicas), como de los factores de presión (como, por ejemplo, las motivaciones económicas y de empleo). Estos resultados exploratorios difieren de las aportaciones previas de Orhan y Scott (2001) o Noorderhaven et al. (2004), quienes defienden el predominio de los factores de atracción sobre los factores de presión para explicar la decisión de convertirse en empresario, en general; y consecuentemente discrepan del argumento expuesto en la Proposición 2. Sin embargo, hay que tomarlos con cautela, dado el carácter exploratorio de este estudio, no siendo posible aportar ningún resultado concluyente en este sentido.

\section{CONCLUSIONES}

Para comprender las motivaciones que explican el emprendimiento social, es necesario tener en cuenta las motivaciones del emprendimiento convencional, pues a pesar de sus evidentes diferencias, ambos tipos de emprendimiento tienen en común algunas de sus principales motivaciones (Dacin et al., 2010).

Sin embargo, el emprendimiento social difiere del emprendimiento convencional en su misión y en cómo poner en marcha el negocio. Las decisiones tomadas en el marco del emprendimiento social no pueden ser valoradas exclusivamente según criterios económicos, ya que podrían considerarse "irracionales". Los emprendedores sociales tienen sus propios valores y motivaciones, lo que les lleva a percibir oportunidades que los emprendedores convencionales no ven como tales (Steinerowski et al., 2008).

Hasta la fecha apenas se han desarrollado investigaciones centradas en las motivaciones específicas para el emprendimiento social (Short, Rana, Bocken y Evans, 2012; Zahra et al., 2009; Dacin et al., 2010). Por ello, este trabajo trata de contribuir a profundizar en el conocimiento de las motivaciones que explican la elección del emprendimiento social como un modelo de negocio. Primero, se ha tratado de identificar estas motivaciones, para después descubrir su nivel de importancia gracias a la colaboración de un grupo de expertos en emprendimiento social y un grupo de emprendedores sociales.

En coherencia con las aportaciones de otros autores (como Dacin et al., 2010), los resultados del estudio muestran que, en 
efecto, emprendimiento social y emprendimiento convencional comparten motivaciones importantes, como la realización personal. Ahora bien, de acuerdo con los argumentos de Steinerowski et al. (2008) o Braga et al. (2014), el emprendimiento social tiene características específicas que lo hacen único y, por lo tanto, motivaciones que también son únicas.

Nuestros resultados indican que los emprendedores sociales son personas que tratan de innovar al servicio del cambio social, es decir, aprovechan su proyecto empresarial para transformar una realidad social imperfecta. Tienen un deseo decidido de transformarse buscando un impacto social y ambiental positivo. Su principal motor son las motivaciones ideológicas, que aseguran su absoluta determinación y convicción de que es posible causar un cambio significativo en la sociedad. Hacer el bien social está en su ADN y sienten que están haciendo algo que les gusta que da sentido a su vida. Es su pasión y sus principios los que los motivan y los alientan a superar las dificultades actuales y las resistencias al cambio social (Curto, 2012).

Las motivaciones económicas y de empleo también son importantes para los emprendedores sociales, de hecho, más de lo que opinan los expertos en emprendimiento social. Ahora bien, los emprendedores sociales son personas de principios que no entran a este mundo atraídas por una posible ayuda financiera o que implementan proyectos de otros que no coinciden con sus sueños. No se mueven por afinidades políticas con el entorno social y tampoco buscan reconocimiento social.

Conocer las valoraciones de estas cuestiones por parte de dos grupos diferentes, expertos y emprendedores sociales, ha servido para enriquecer los resultados, al poner de relieve discrepancias entre las valoraciones de ambos grupos que invitan a un estudio adicional. Hemos visto que, si bien las motivaciones ideológicas son claramente más importantes para los emprendedores sociales, los expertos sitúan en primer lugar las motivaciones relacionadas con la realización personal. En consecuencia, los emprendedores sociales se sienten más capaces y cualificados de lo que reconocen los expertos.

En este sentido, los resultados de este estudio pueden tener implicaciones de interés, tanto para los emprendedores sociales como para las entidades públicas y privadas que tratan de 
prestar apoyo a este tipo de iniciativas. Para los emprendedores sociales, pueden servir para reflexionar acerca de las competencias y capacidades necesarias para liderar este tipo de proyectos empresariales. Es importante que no se dejen llevar por su pasión por la misión social, o que esto les impida ser realmente conscientes de sus limitaciones y/o carencias. Con relación a las entidades, tanto públicas como privadas, que ofrecen recursos de apoyo al emprendimiento social, deben también hacer una reflexión profunda sobre su conocimiento de los emprendedores sociales y sus necesidades.

Finalmente, la principal limitación de este trabajo es su naturaleza exploratoria y el tamaño limitado de la muestra. Si bien las valoraciones de este grupo de expertos y emprendedores sociales pueden ayudar a comprender mejor las motivaciones para comenzar y tratar de sacar adelante un proyecto de emprendimiento social, los resultados no pueden generalizarse. En consecuencia, para concluir se sugieren posibles líneas de investigación futuras. En primer lugar, se debe realizar un estudio de estos temas desde varias perspectivas para obtener una visión más fiel de la situación que se está estudiando. En segundo lugar, sería interesante incorporar nuevas variables, por ejemplo, el ciclo de vida del proyecto para descubrir cómo pueden cambiar estas motivaciones a medida que avanza el proyecto de emprendimiento social. Por último, todos estos análisis deben completarse con estudios confirmatorios.

\section{BIBLIOGRAFÍA}

Amit, R. y Muller, E. (1995). Push and pull entrepreneurship (two types based on motivation, Journal of Small Business and Entrepreneurship, Vol. 12, No. 4, pp. 64-80.

Austin, J., Stevenson, H. y Wei-Skillern, J. (2006). Social and commercial entrepreneurship: Same, different, or both? Entrepreneurship Theory and Practice, Vol. 30, No. 1, pp. 1-22.

Boschee, J. (2008). Social Entrepreneurship: The Promise and the Perils, in A. Nicholls (ed.), Social entrepreneurship: New models of sustainable social change (pp. 356-390). OUP, Oxford. 
Braga, J.C., Proença, T. y Ferreira, M.R. (2014). Motivations for social entrepreneurship. Evidences from Portugal, Tékhne, Vol. 12, pp.11-21.

CEPES. (2018). Las empresas más relevantes de la Economía Social 2017-18. https://www.cepes.es/publicaciones

Cools, E. y Vermeulen, S. (2008). What's in a name? An inquiry on the cognitive and entrepreneurial profile of the social entrepreneur (No. 2008-02). Vlerick Leuven Gent Management School.

Curto Grau, M. (2012). Los emprendedores sociales: innovación al servicio del cambio social, Cuadernos de la Cátedra La Caixa de la Responsabilidad Social de la Empresa y Gobierno Corporativo, No. 13, pp. 1-23.

Dacin, P.A., Dacin, M.T. y Matear, M. (2010). Social entrepreneurship: Why we don't need a new theory and how we move forward from here, Academy of Management Perspectives, Vol. 24, No. 2, pp. 36-56.

Dacin, M. T., Dacin, P. A., y Tracey, P. (2011). Social entrepreneurship: A critique and future directions. Organization science, 22(5), 1203-1213.

Dwivedi, A. y Weerawardena, J. (2018). Conceptualizing and operationalizing the social entrepreneurship construct. Journal of Business Research, 86, 32-40.

Fayolle, A. y Liñán, F. (2014). The future of research on entrepreneurial intentions. Journal of Business Research, 67(5), 663-666.

Fernández-Ballesteros, R. (1995). Evaluación de programas: una guía práctica en ámbitos sociales, educativos y de salud. Editorial Síntesis, Madrid.

García-Ruiz, M. E. y Lena-Acebo, F. J. (2018). Aplicación del metodo delphi en el diseño de una investigación cuantitativa sobre el fenómeno FABLAB. Empiria. Revista de metodología de ciencias sociales, (40), 129-166.

Gilad, B. y Levine, P. (1986). A behavioral model of entrepreneurial supply, Journal of Small Business Management, Vol. 24, No. 4, pp. 45-54.

Hall, K., Miller, R. y Millar, R. (2012). Jumped or pushed: What motivates NHS staff to set up a social enterprise? Social Enterprise Journal, Vol. 8, pp. 49-62. 
Kirkwood, J. (2009). Motivational factors in a push-pull theory of entrepreneurship, Gender in Management: An International Journal, Vol. 24, No. 5, pp. 346-364.

Kirkwood, J. y Walton, S. (2010). What motivates ecopreneurs to start businesses? International Journal of Entrepreneurial, Behavior \& Research, Vol. 16, No. 3, pp. 204-228.

Landeta, J. (2002). El método Delphi: una técnica de previsión del futuro. Ariel, Barcelona.

Lannoy, A. y Porcaccia, M. (2001). L'utilisation du jugement d'experts en sûreté de fonctionnement. Editions TEC \& DOC, Paris.

Latham, G. P. y Pinder, C. C. (2005). Work motivation theory and research at the dawn of the twenty-first century, Annual Review of Psychology, Vol. 56, pp. 485-516.

Locke, E. A. (2000). Motivation, cognition, and action: An analysis of studies of task goals and knowledge, Applied Psychology: An International Review, Vol. 49, No. 3, 408-429.

Mair, J. y Martí, I. (2006). Social entrepreneurship research: A source of explanation, prediction, and delight, Journal of World Business, Vol. 41, No. 1, pp. 36-44.

Mair, J. y Noboa, E. (2005). How intentions to create a social venture are formed. A case study (No. D/593). IESE Business School.

Marques, C., Ferreira, J., Ferreira, F. y Lages, M. (2012). Entrepreneurial orientation and motivation to start up a business: Evidence from the health service industry, Entrepreneurship Management Journal, Vol. 9, No. 1, pp 77-94.

Martínez, E. (2003). La Técnica Delphi como estrategia de consulta a los implicados en la evaluación de programas. Revista de Investigación Educativa, Vol. 21, No. 2, pp. 449-163.

Miller, T., Grimes, M., McMullen, J. y Vogus, T. (2012). Venturing for others with heart and head: How compassion encourages social entrepreneurship, Academy of Management Review, Vol. 37, No. 4, pp. 616-640.

Noorderhaven, N., Thurik, R., Wennekers, S. y van Stel, A. (2004). The role of dissatisfaction and per capita income in explaining 
self-employment across 15 European countries, Entrepreneurship Theory and Practice, Vol. 28, pp. 447-466.

Orhan, M. Scott, D. (2001). Why women enter into entrepreneurship: an explanatory model, Women in Management Review, Vol. 16, No. 5, pp. 232-43.

Pless, N.M. (2012). Social entrepreneurship in theory and practice-an introduction, Journal of Business Ethics, Vol. 111, No. 3, pp. 317-320.

Prabhu, G. N. (1999). Social entrepreneurial leadership. Career Development International, Vol. 4, No. 3, pp. 140-145.

Rey-Martí, A., Ribeiro-Soriano, D. y Sánchez-García, J. L. (2016). Giving back to society: Job creation through social entrepreneurship. Journal of Business Research, 69(6), 2067-2072.

Romero, R., Cabero, J., Llorente M. C. y Vázquez-Martínez, A. I. (2012). El método Delphi y la formación del profesorado en TIC. Global, 9, (44), 81-92.

Rowe, G., y Wright, G. (1999). The Delphi technique as a forecasting tool: issues and analysis. International Journal of Forecasting, 15, 353-375.

Scheibe, M., Skutsch, M. y Schofer, J. (1975). Experiments in Delphi methodology. En H. A. Linstone y M. Turoff (Eds.), The Delphi method: Techniques and applications.

Schjoedt, L. y Shaver, K.G. (2007). Deciding on an entrepreneurial career: a test of the pull and push hypotheses using the panel study of entrepreneurial dynamics data, Entrepreneurship Theory \& Practice, Vol. 31, No. 5, pp. 733-52.

Segal, G., Borgia, D. y Schoenfeld, J. (2005). The motivation to become an entrepreneur, International Journal of Entrepreneurial Behaviour \& Research, Vol. 11, No. 1, pp. 42-57.

Shaw, E. y Carter, S. (2007). Social entrepreneurship: Theoretical antecedents and empirical analysis of entrepreneurial processes and outcomes, Journal of Small Business and Enterprise Development, Vol. 14, No. 3, pp. 418-434.

Short, S. W., Rana, P., Bocken, N. M. y Evans, S. (2012). Embedding sustainability in business modelling through multistakeholder value innovation. E IFIP International Conference 
on Advances in Production Management Systems (pp. 175183). Springer Berlin Heidelberg.

Steinerowski, A., Jack, S. y Farmer, J. (2008). Who are the social entrepreneurs and what do they actually do? En Babson College Entrepreneurship Research Conference BCERC. Babson College Centre for Entrepreneurship.

Thompson, J.L. (2002). The world of the social entrepreneur, International Journal of Public Sector Management, Vol. 15, $\mathrm{N}^{\circ}$. 5, pp. 412-431.

Uceda, M. E. G., Luna, J. L. M., y Lafuente, J. A. (2017). Application of the Delphi method for the analysis of the factors determining social entrepreneurship. Journal of Business, 9(1), 43-66.

Weerawardena, J. y Sullivan Mort, G. (2006). Investigating social entrepreneurship: A multidimensional model, Journal of World Business, Vol. 41, No. 1, pp. 21-35.

Zahra, S.A., Rawhouser, H.N., Bhawe, N., Neubaum, D.O. y Hayton, J.C. (2008). Globalization of social entrepreneurship opportunities, Strategic Entrepreneurship Journal, Vol. 2, No. 2, pp. 117-131. 